LYCEN 9307

May 1993

\title{
Sum Rules for Multi-Photon Spectroscopy of Ions in Finite Symmetry
}

\author{
M. KIBLER and M. DAOUD \\ Institut de Physique Nucléaire, IN2P3 - CNRS et Université Lyon-1, \\ 43 Boulevard du 11 Novembre 1918, 69622 Villeurbanne Cedex, France
}

\begin{abstract}
Models describing one- and two-photon transitions for ions in crystalline environments are unified and extended to the case of parity-allowed and parity-forbidden $p$-photon transitions. The number of independent parameters for characterizing the polarization dependence is shown to depend on an ensemble of properties and rules which combine symmetry considerations and physical models.
\end{abstract}

Published in Letters in Mathematical Physics 28 (1993) 269-280. 


\section{Introduction and Preliminaries}

Electronic spectroscopy of partly-filled shell ions, like $d^{N}$ and $f^{N}$ ions, embedded in condensed matter surroundings has been the object of important developments in the recent years. In particular, the advent of tunable dye lasers has made possible to perform twophoton spectroscopy experiments. This Letter is concerned with $p$-photon spectroscopy of a transition ion with ground atomic configuration $n \ell^{N}$ in a given crystal field with generic symmetry $G$. We address here the question of obtaining a closed-form expression for the intensity of a $p$-photon (absorption) transition between an initial Stark level $i$ with symmetry species $\Gamma$ and a final Stark level $f$ with symmetry species $\Gamma^{\prime}$. The labels $\Gamma$ and $\Gamma^{\prime}$ stand for two IRC's (irreducible representation classes) of the double group $G^{*}$ of $G \simeq G^{*} / Z_{2}$. The group $G^{*}$ may be considered (up to isomorphism) as a subgroup of $S U(2)$.

According to Dirac and Göppert-Mayer, the transition moment $M_{i(\Gamma \gamma) \rightarrow f\left(\Gamma^{\prime} \gamma^{\prime}\right)}$ for a p-photon transition between the initial state $i(\Gamma \gamma)$ and the final state $f\left(\Gamma^{\prime} \gamma^{\prime}\right)$ is given by

$$
M_{i(\Gamma \gamma) \rightarrow f\left(\Gamma^{\prime} \gamma^{\prime}\right)}=\sum_{v_{j}} \frac{\left(f \Gamma^{\prime} \gamma^{\prime}\left|\left(\mathbf{D} . \mathbf{E}_{1}\right)\right| v_{1}\right)\left(v_{1}\left|\left(\mathbf{D} . \mathbf{E}_{2}\right)\right| v_{2}\right) \cdots\left(v_{p-1}\left|\left(\mathbf{D} . \mathbf{E}_{p}\right)\right| i \Gamma \gamma\right)}{\left(\Omega_{i}-\Omega_{v_{1}}\right)\left(\Omega_{i}-\Omega_{v_{2}}\right) \cdots\left(\Omega_{i}-\Omega_{v_{p-1}}\right)}+\text { perm }
$$

in the framework of the electric dipolar approximation. The sum on $v_{j}$ in $(1)$ has to be performed on all the intermediate states and "perm" indicates that $p$ ! permuted terms arise in the Feynman representation associated to $M_{i(\Gamma \gamma) \rightarrow f\left(\Gamma^{\prime} \gamma^{\prime}\right)}$ when the $p$ photons are different. The operator $\left(\mathbf{D} . \mathbf{E}_{k}\right)$ in (1) is the scalar product of the dipole moment operator $\mathbf{D}$ for the $N$ electrons with the unit polarization vector $\mathbf{E}_{k}$ of the single-mode for the $k$ th photon. (We use single-modes to describe the electromagnetic field.) Furthermore, the factors $\left(\Omega_{i}-\Omega_{v_{j}}\right)$ are energy denominators for the system radiation field plus ion in its environment. Finally, the labels $\gamma$ and $\gamma^{\prime}$ distinguish the components of the Stark levels $i$ and $f$ when the dimensions $[\Gamma]$ and $\left[\Gamma^{\prime}\right]$ of the IRC's $\Gamma$ and $\Gamma^{\prime}$ are greater than 1 , respectively. 
At this stage, we must consider two types of $p$-photon transitions: the parity-allowed and parity-forbidden transitions. The parity-allowed transitions occur either between levels of the same parity $\left(i \in n \ell^{N}\right.$ and $\left.f \in n \ell^{N}\right)$ when $p$ is even or between levels of opposite parities $\left(i \in n \ell^{N}\right.$ and $f \in n \ell^{N-1} n^{\prime} \ell^{\prime}$ with $\left.(-1)^{\ell+\ell^{\prime}}=-1\right)$ when $p$ is odd. The parityforbidden transitions $\left(n \ell^{N} \rightarrow n \ell^{N}\right.$ for $p$ odd and $n \ell^{N} \rightarrow n \ell^{N-1} n^{\prime} \ell^{\prime}$ with $(-1)^{\ell+\ell^{\prime}}=-1$ for $p$ even) may become possible, via the Van Vleck mechanism, if $G$ does not have a center of inversion (or if the inversion symmetry is broken by vibronic coupling or ligand polarization).

The sums over the intermediate states $v_{j}$ in (1) can be effectuated by using a quasiclosure approximation of the type of the ones used by many authors [1-5] for $p=1$ or 2 in the cases of $d^{N}$ and $f^{N}$ ions. Such an approximation used in conjunction with recoupling techniques for $S U(2)$ irreducible tensorial sets leads to

$$
M_{i(\Gamma \gamma) \rightarrow f\left(\Gamma^{\prime} \gamma^{\prime}\right)}=\left(f \Gamma^{\prime} \gamma^{\prime}\left|H_{\mathrm{eff}}\right| i \Gamma \gamma\right)
$$

where the model-dependent operator $H_{\text {eff }}$ is an effective operator described in Section 2 .

Both for parity-allowed and parity-forbidden transitions, we take the initial and final state vectors in the symmetry adapted form $(x \equiv i$ or $f)$

$$
\left.\mid x \Gamma \gamma)=\sum_{\alpha S L J a} \mid \alpha S L J a \Gamma \gamma\right) c(\alpha S L J a \Gamma ; x)
$$

The initial state $i(\Gamma \gamma)$ belongs to the configuration $n \ell^{N}$ and the final state $f\left(\Gamma^{\prime} \gamma^{\prime}\right)$ belongs either to the configuration $n \ell^{N}$ or to the configuration $n \ell^{N-1} n^{\prime} \ell^{\prime}$. In (3), the $S U(2) \supset G^{*}$ vectors of type $\mid j a \Gamma \gamma)$ are connected to the $S U(2) \supset U(1)$ vectors of type $\mid j m)$ by

$$
\left.\mid j a \Gamma \gamma):=\sum_{m=-j}^{j} \mid j m\right)(j m \mid j a \Gamma \gamma)
$$

The branching label $a$ in (4) has to be used when the IRC $\Gamma$ of $G^{*}$ occurs several times in the IRC $(j)$ of $S U(2)$. This external multiplicity label may be described, at least partially, 
by IRC's of subgroups of $S U(2)$ that contain in turn the group $G^{*}$. The expansions (3) and (4) are of a completely different nature. Equation (4) is of a group theoretical nature: the reduction coefficients $(j m \mid j a \Gamma \gamma)$ depend on the chain $S U(2) \supset G^{*}$ but not on the physics of the problem. On the other hand, the expansions (3) depend on physical models: the $c$ coefficients may be obtained from the diagonalization of one or two $G$-invariant Hamiltonians (according to whether as the initial and final configurations are the same or different) describing the ion in its environment. It is to be noted that, thanks to Schur's lemma, we may always standardize the reduction coefficients $(j m \mid j a \Gamma \gamma)$ in (4) in such a way that the expansion coefficients $c$ in (3) be independent of the generalized magnetic quantum numbers $\gamma$ and $\gamma^{\prime}$. This result is essential in view of the sum on $\gamma$ and $\gamma^{\prime}$ in (5) below. Equation (3) indicates that we adopt here the philosophy of [6-9] where the diagonalization-optimization of the $G$-invariant Hamiltonian(s) is done, without loss of generality, in a weak-field basis adapted to the chain $S U(2) \supset G^{*}$ : the labels $\alpha, S, L$ and $J$ are not taken a priori as good quantum numbers; the only good quantum numbers are $\Gamma, \gamma$ and $\Gamma^{\prime}, \gamma^{\prime}$ for the initial and final state vectors, respectively (and the parity for parity-allowed transitions).

The physical quantity we want to calculate in Section 2 is the intensity

$$
S_{i(\Gamma) \rightarrow f\left(\Gamma^{\prime}\right)}:=\sum_{\gamma \gamma^{\prime}}\left|M_{i(\Gamma \gamma) \rightarrow f\left(\Gamma^{\prime} \gamma^{\prime}\right)}\right|^{2}
$$

for the $i(\Gamma) \rightarrow f\left(\Gamma^{\prime}\right)$ transition. The sum in (5) has to be done over all the external multiplicity labels $\gamma$ and $\gamma^{\prime}$, i.e., over all the components of the initial and final Stark levels. (The labels $\gamma$ and $\gamma^{\prime}$ may be characterized, at least partially, by IRC's of subgroups of $G^{*}$.) The purpose of this work is to show how far group theory can go in the determination of $S_{i(\Gamma) \rightarrow f\left(\Gamma^{\prime}\right)}$ in connection with sophisticated models for the operator $H_{\mathrm{eff}}$ of $(2)$. In particular, we want to clearly separate the elements arising from group theory (via WignerRacah calculus) from the model-dependent quantities (both for the light-matter interaction 
and the description of the $\ell^{N}$ ion in its environment). Rather than considering $G^{*}$ as an isolated group, we shall consider it as a subgroup of $S U(2)$, an approach that has already proved to be very useful as far as the energy spectrum of the $\ell^{N}$ ion in $G$ symmetry is concerned [8,9]. The basic tool is thus the Wigner-Racah algebra of the group $G^{*}$ in a $G^{*} \subset S U(2)$ basis. Therefore we develop, in Section 1, those nontrivial aspects of the Wigner-Racah algebra for the chain $S U(2) \supset G^{*}$ that are essential for performing the sum in (5). The corollary obtained in Section 1 is applied in Section 2 to derive the sum rule (23) with the accompanying properties and rules (26-28).

\section{Sum Rule for $S U(2) \supset G^{*}$ Coupling Coefficients}

Equation (4) defines the $\{j a \Gamma \gamma\}$ scheme for the chain $S U(2) \supset G^{*}$ that is more appropriate in condensed matter spectroscopy than the $\{j m\}$ scheme for the chain $S U(2) \supset U(1)[6,8,9]$. For $j, a$ and $\Gamma$ fixed, the set $\{\mid j a \Gamma \gamma): \gamma=1, \cdots,[\Gamma]\}$ is a (standardized) $G$-irreducible tensorial set (in the sense of Fano and Racah) of vectors associated to $\Gamma$. Similarly, from

the $S U(2) \supset U(1)$ spherical tensor operators $U_{q}^{(k)}$, we define the operators $U_{a \Gamma \gamma}^{(k)}$ by an expansion similar to (4) so that, for $k, a$ and $\Gamma$ fixed, the set $\left\{U_{a \Gamma \gamma}^{(k)}: \gamma=1, \cdots,[\Gamma]\right\}$ is a $G$-irreducible tensorial set of operators associated to $\Gamma$. The latter $G$-irreducible tensorial sets are also labelled by IRC's of $S U(2)$ and, therefore, we can easily generate, by direct sum, nonstandard $S U(2)$-irreducible tensorial sets. Thus, we may apply the Wigner-Eckart theorem for the group $S U(2)$ in a nonstandard basis adapted to its subgroup $G^{*}$. As a result, we have [8]

$$
\begin{aligned}
& \left(\tau_{1} j_{1} a_{1} \Gamma_{1} \gamma_{1}\left|U_{a \Gamma \gamma}^{(k)}\right| \tau_{2} j_{2} a_{2} \Gamma_{2} \gamma_{2}\right)=\left(\tau_{1} j_{1}\left\|U^{(k)}\right\| \tau_{2} j_{2}\right) \\
& \sum_{a_{3} \Gamma_{3} \gamma_{3}}\left(\begin{array}{ccc}
j_{1} & \\
& & \\
a_{3} \Gamma_{3} \gamma_{3} & & a_{1} \Gamma_{1} \gamma_{1}
\end{array}\right) \bar{f}\left(\begin{array}{ccc}
j_{1} & k & j_{2} \\
a_{3} \Gamma_{3} \gamma_{3} & a \Gamma \gamma & a_{2} \Gamma_{2} \gamma_{2}
\end{array}\right)^{*}
\end{aligned}
$$


with

$$
\begin{array}{r}
\bar{f}\left(\begin{array}{ccc}
j_{1} & j_{2} & j_{3} \\
a_{1} \Gamma_{1} \gamma_{1} & a_{2} \Gamma_{2} \gamma_{2} & a_{3} \Gamma_{3} \gamma_{3}
\end{array}\right):=\sum_{m_{1} m_{2} m_{3}}\left(\begin{array}{ccc}
j_{1} & j_{2} & j_{3} \\
m_{1} & m_{2} & m_{3}
\end{array}\right) \\
\left(j_{1} m_{1} \mid j_{1} a_{1} \Gamma_{1} \gamma_{1}\right)^{*}\left(j_{2} m_{2} \mid j_{2} a_{2} \Gamma_{2} \gamma_{2}\right)^{*}\left(j_{3} m_{3} \mid j_{3} a_{3} \Gamma_{3} \gamma_{3}\right)^{*}
\end{array}
$$

and

$$
\left(\begin{array}{ccc} 
& j & \\
& & \\
a_{1} \Gamma_{1} \gamma_{1} & & a_{2} \Gamma_{2} \gamma_{2}
\end{array}\right):=[j]^{1 / 2} \bar{f}\left(\begin{array}{ccc}
j & 0 & j \\
a_{1} \Gamma_{1} \gamma_{1} & a_{0} \Gamma_{0} \gamma_{0} & a_{2} \Gamma_{2} \gamma_{2}
\end{array}\right),
$$

where $\Gamma_{0}$ denotes the identity IRC of $G^{*}$. In the right hand side of $(6)$, the quantum numbers $\tau_{1}$ and $\tau_{2}$, external to the chain $S U(2) \supset G^{*}$, appear in the reduced matrix element.

The $\bar{f}$ or $3-j a \Gamma \gamma$ symbol in (7) is an $S U(2) \supset G^{*}$ symmetry adapted form of the usual 3-jm Wigner symbol. It constitutes a symmetrized form of the coefficient [8]

$$
\begin{aligned}
f\left(\begin{array}{ccc}
j_{1} & j_{2} & k \\
a_{1} \Gamma_{1} \gamma_{1} & a_{2} \Gamma_{2} \gamma_{2} & a \Gamma \gamma
\end{array}\right):= & \sum_{m_{1} q m_{2}}(-1)^{j_{1}-m_{1}}\left(\begin{array}{ccc}
j_{1} & k & j_{2} \\
-m_{1} & q & m_{2}
\end{array}\right) \\
& \left(j_{1} m_{1} \mid j_{1} a_{1} \Gamma_{1} \gamma_{1}\right)^{*}(k q \mid k a \Gamma \gamma)\left(j_{2} m_{2} \mid j_{2} a_{2} \Gamma_{2} \gamma_{2}\right)
\end{aligned}
$$

that generalizes the $f$ coefficient defined by Racah and some of his students (in particular Schönfeld and Flato, see references $[6,7])$.

The 2-ja $\gamma \gamma$ symbol defined by (8) turns out to be an $S U(2) \supset G^{*}$ symmetry adapted form of the Herring-Wigner metric tensor. Indeed, from (7) and (8) we have

$$
\left(\begin{array}{ccc} 
& j & \\
& & \\
a_{1} \Gamma_{1} \gamma_{1} & & a_{2} \Gamma_{2} \gamma_{2}
\end{array}\right)=\sum_{m}(-1)^{j+m}\left(j m \mid j a_{1} \Gamma_{1} \gamma_{1}\right)^{*}\left(j,-m \mid j a_{2} \Gamma_{2} \gamma_{2}\right)^{*} .
$$

Therefore, the metric tensor given by (10) allows us to handle all the phases occurring in the $\{j a \Gamma \gamma\}$ scheme $[8]$.

By combining (7), (9) and (10), we can rewrite (6) in the simple form

$$
\left(\tau_{1} j_{1} a_{1} \Gamma_{1} \gamma_{1}\left|U_{a \Gamma \gamma}^{(k)}\right| \tau_{2} j_{2} a_{2} \Gamma_{2} \gamma_{2}\right)=\left(\tau_{1} j_{1}\left\|U^{(k)}\right\| \tau_{2} j_{2}\right) \quad f\left(\begin{array}{ccc}
j_{1} & j_{2} & k \\
a_{1} \Gamma_{1} \gamma_{1} & a_{2} \Gamma_{2} \gamma_{2} & a \Gamma \gamma
\end{array}\right)
$$


The interest of (6) and (11) for electronic spectroscopy of ions in crystalline fields has been discussed in $[8,9]$. From a mathematical viewpoint, it is to be observed that the factorization in (11) into the product of a $G^{*}$-dependent factor (the $f$ coefficient) by a $G^{*}$-independent factor is valid whatsoever the group $G^{*}$ is multiplicity free or not. The internal multiplicity problem arising when $G^{*}$ is not multiplicity free is thus easily solved by making use of (11).

The $f$ coefficients may be considered as the components of a third-rank tensor (secondrank covariant and first-rank contravariant). In contradistinction, the $\bar{f}$ symbol defines a third-rank contravariant tensor. The other properties of the $\bar{f}$ and $f$ coefficients that are of relevance for this work are based on the following lemma.

LEMMA (Racah's lemma). We have the factorization formula [10]

$$
\begin{aligned}
f\left(\begin{array}{ccc}
j_{1} & j_{2} & k \\
a_{1} \Gamma_{1} \gamma_{1} & a_{2} \Gamma_{2} \gamma_{2} & a \Gamma \gamma
\end{array}\right)= & (-1)^{2 k}\left[j_{1}\right]^{-1 / 2} \\
& \sum_{\beta}\left(j_{2} a_{2} \Gamma_{2}+k a \Gamma \mid j_{1} a_{1} \beta \Gamma_{1}\right)^{*}\left(\Gamma_{2} \Gamma \gamma_{2} \gamma \mid \Gamma_{2} \Gamma \beta \Gamma_{1} \gamma_{1}\right)^{*},
\end{aligned}
$$

where $\left(j_{2} a_{2} \Gamma_{2}+k a \Gamma \mid j_{1} a_{1} \beta \Gamma_{1}\right)$ is an isoscalar factor or reduced $f$ symbol (independent of the quantum numbers $\gamma_{1}, \gamma_{2}$ and $\left.\gamma\right)$ and $\left(\Gamma_{2} \Gamma \gamma_{2} \gamma \mid \Gamma_{2} \Gamma \beta \Gamma_{1} \gamma_{1}\right)$ a Clebsch-Gordan coefficient for the group $G^{*}$ considered as an isolated entity. (The label $\beta$ is an internal multiplicity label to be used when the Kronecker product $\Gamma_{2} \otimes \Gamma$ is not multiplicity free.)

Proof. Equation (12) is a consequence of Schur's lemma.

In order to be able to use tables and/or computer programs for the highly symmetrical $\bar{f}$ symbol (and for the $R_{\beta}$ or reduced $\bar{f}$ symbol, see (13) and (14)), it is useful to transcribe 
(12) for the $\bar{f}$ symbol. We thus obtain

$\bar{f}\left(\begin{array}{ccc}j_{1} & j_{2} & j_{3} \\ a_{1} \Gamma_{1} \gamma_{1} & a_{2} \Gamma_{2} \gamma_{2} & a_{3} \Gamma_{3} \gamma_{3}\end{array}\right)=\sum_{\beta} R_{\beta}\left(\begin{array}{ccc}j_{1} & j_{2} & j_{3} \\ a_{1} \Gamma_{1} & a_{2} \Gamma_{2} & a_{3} \Gamma_{3}\end{array}\right) V_{\beta}\left(\begin{array}{ccc}\Gamma_{1} & \Gamma_{2} & \Gamma_{3} \\ \gamma_{1} & \gamma_{2} & \gamma_{3}\end{array}\right)$,

where $V_{\beta}$ is an extension, taking into account the multiplicity label $\beta$, of the $V$ coefficient introduced by Griffith [11]. The coefficient

$$
R_{\beta}\left(\begin{array}{ccc}
j_{1} & j_{2} & j_{3} \\
a_{1} \Gamma_{1} & a_{2} \Gamma_{2} & a_{3} \Gamma_{3}
\end{array}\right) \equiv R_{\beta}\left(\begin{array}{ccc}
j_{1} & j_{2} & j_{3} \\
a_{1} \Gamma_{1} & a_{2} \Gamma_{2} & a_{3} \Gamma_{3}
\end{array}\right)_{G^{*}}^{S U(2)}
$$

characterizes the restriction from $S U(2)$ to $G^{*}$; it is a generalization of the $V$ symbol introduced by the Chinese school of Tang Au-chin [12]. (In (13) and (14), the label $\beta$ is absolutely necessary when the identity IRC $\Gamma_{0}$ appears several times in $\Gamma_{1} \otimes \Gamma_{2} \otimes \Gamma_{3}$.) The $R_{\beta}$ symbol is connected to the isoscalar factor of (12) by

$$
\begin{aligned}
\left(j_{2} a_{2} \Gamma_{2}+k a \Gamma \mid j_{1} a_{1} \beta_{1} \Gamma_{1}\right)= & (-1)^{2 k}\left[\Gamma_{1}\right]^{-1}\left[j_{1}\right] \sum_{a_{3} \Gamma_{3}} \sum_{\beta_{2}} U\left(\Gamma_{1} \Gamma_{2} \Gamma\right)_{\beta_{1} \beta_{2}} \\
& R_{\beta_{2}}\left(\begin{array}{ccc}
k & j_{2} & j_{1} \\
a \Gamma & a_{2} \Gamma_{2} & a_{3} \Gamma_{3}
\end{array}\right) R\left(\begin{array}{ccc}
0 & j_{1} & j_{1} \\
& & \\
a_{0} \Gamma_{0} & a_{1} \Gamma_{1} & a_{3} \Gamma_{3}
\end{array}\right)^{*},
\end{aligned}
$$

where $U\left(\Gamma_{1} \Gamma_{2} \Gamma\right)$ is an arbitrary unitary matrix. Indeed, this matrix is a simple phase factor when the Kronecker product $\Gamma_{2} \otimes \Gamma$ is multiplicity free.

Repeated applications of Racah's lemma and of the orthonormality-completeness property for the Clebsch-Gordan coefficients of $G^{*}$ lead to the following corollary.

COROLLARY. The sum

$$
X:=\sum_{\gamma_{1} \gamma_{2}} f\left(\begin{array}{ccc}
j_{1} & j_{2} & k_{3} \\
a_{1} \Gamma_{1} \gamma_{1} & a_{2} \Gamma_{2} \gamma_{2} & a_{3} \Gamma_{3} \gamma_{3}
\end{array}\right)^{*} f\left(\begin{array}{ccc}
j_{4} & j_{5} & k_{6} \\
a_{4} \Gamma_{1} \gamma_{1} & a_{5} \Gamma_{2} \gamma_{2} & a_{6} \Gamma_{6} \gamma_{6}
\end{array}\right)
$$


is diagonal in $\left(\Gamma_{3}, \Gamma_{6}\right)$ and $\left(\gamma_{3}, \gamma_{6}\right)$. Furthermore, the diagonal value of $X$ is independent of $\gamma_{3}$. More precisely, we have

$$
\begin{array}{r}
X=\delta\left(\Gamma_{6}, \Gamma_{3}\right) \delta\left(\gamma_{6}, \gamma_{3}\right)(-1)^{2\left(k_{3}+k_{6}\right)}\left(\left[j_{1}\right]\left[j_{4}\right]\right)^{-1 / 2}\left[\Gamma_{3}\right]^{-1}\left[\Gamma_{1}\right] \\
Z\left(j_{1} a_{1}, j_{2} a_{2}, k_{3} a_{3}, j_{4} a_{4}, j_{5} a_{5}, k_{6} a_{6} ; \Gamma_{1} \Gamma_{2} \Gamma_{3}\right),
\end{array}
$$

where

$$
\begin{aligned}
& Z\left(j_{1} a_{1}, j_{2} a_{2}, k_{3} a_{3}, j_{4} a_{4}, j_{5} a_{5}, k_{6} a_{6} ; \Gamma_{1} \Gamma_{2} \Gamma_{3}\right):= \\
& \sum_{\beta}\left(j_{2} a_{2} \Gamma_{2}+k_{3} a_{3} \Gamma_{3} \mid j_{1} a_{1} \beta \Gamma_{1}\right)\left(j_{5} a_{5} \Gamma_{2}+k_{6} a_{6} \Gamma_{3} \mid j_{4} a_{4} \beta \Gamma_{1}\right)^{*}
\end{aligned}
$$

that reduces to the product of two isoscalar factors when $G^{*}$ is multiplicity free.

It is also useful to express $Z$ and thus $X$ in terms of the $R_{\beta}$ symbols which are more symmetrical than the isoscalars factors. In this respect, by using (15) we can write (18) as

$$
\begin{array}{r}
Z\left(j_{1} a_{1}, j_{2} a_{2}, k_{3} a_{3}, j_{4} a_{4}, j_{5} a_{5}, k_{6} a_{6} ; \Gamma_{1} \Gamma_{2} \Gamma_{3}\right)=(-1)^{2\left(k_{3}+k_{6}\right)}\left[\Gamma_{1}\right]^{-2}\left[j_{1}\right]\left[j_{4}\right] \\
\sum_{\beta} \sum_{a_{x} \Gamma_{x}} \sum_{a_{y} \Gamma_{y}} R\left(\begin{array}{ccc}
0 & j_{1} & j_{1} \\
& & \\
a_{0} \Gamma_{0} & a_{1} \Gamma_{1} & a_{x} \Gamma_{x}
\end{array}\right)^{*} R_{\beta}\left(\begin{array}{ccc}
k_{3} & j_{2} & j_{1} \\
& & \\
a_{3} \Gamma_{3} & a_{2} \Gamma_{2} & a_{x} \Gamma_{x}
\end{array}\right)^{*} \\
R\left(\begin{array}{ccc}
0 & j_{4} & j_{4} \\
a_{0} \Gamma_{0} & a_{4} \Gamma_{1} & a_{y} \Gamma_{y}
\end{array}\right)^{*} R_{\beta}\left(\begin{array}{ccc}
k_{6} & j_{5} & j_{4} \\
a_{6} \Gamma_{3} & a_{5} \Gamma_{2} & a_{y} \Gamma_{y}
\end{array}\right)^{*}
\end{array}
$$

that is in fact independent of the $U$ matrix of Equation (15).

\section{Intensity Formula}

For a given transition operator $H_{\text {eff }}$, we are now in a position to effectuate the summation over $\gamma$ and $\gamma^{\prime}$ in (5) and, thus, to obtain a compact expression for the intensity $S_{i(\Gamma) \rightarrow f\left(\Gamma^{\prime}\right)}$. A very general expression for the effective operator $H_{\text {eff }}$ is

$$
H_{\mathrm{eff}}=\sum_{k_{1} k_{2} \cdots k_{p-1}} \sum_{t} \sum_{k_{S} k_{L}} \sum_{k} C\left[k_{1} k_{2} \cdots k_{p-1} ; t ; k_{S} k_{L} ; k\right]\left(\left\{\mathbf{P}^{\left(k_{p-1}\right)} \mathbf{X}^{(t)}\right\}^{(k)} \cdot \mathbf{W}^{\left(k_{S} k_{L}\right) k}\right),
$$


where $($.$) is a scalar product involving electronic \left(\mathbf{W}^{\left(k_{S} k_{L}\right) k}\right)$ and nonelectronic $\left(\mathbf{P}^{\left(k_{p-1}\right)}\right.$ and $\left.\mathbf{X}^{(t)}\right)$ tensors. In Equation $(20), \mathbf{P}^{\left(k_{p-1}\right)}$ denotes the polarization tensor defined by

$$
\mathbf{P}^{\left(k_{p-1}\right)}:=\left\{\cdots\left\{\left\{\mathbf{E}_{1} \mathbf{E}_{2}\right\}^{\left(k_{1}\right)} \mathbf{E}_{3}\right\}^{\left(k_{2}\right)} \cdots \mathbf{E}_{p}\right\}^{\left(k_{p-1}\right)}
$$

that describes the coupling of the $p$ polarization vectors and that is entirely under the control of the experimentalist. The tensors $\mathbf{W}^{\left(k_{S} k_{L}\right) k}$ and $\mathbf{X}^{(t)}$ are relative to the ion and its environment: $\mathbf{W}^{\left(k_{S} k_{L}\right) k}$ is an electronic double tensor of spin degree $k_{S}$, orbital degree $k_{L}$ and total degree $k$ whereas $\mathbf{X}^{(t)}$ is a (single) tensor of the degree $t$, for instance, the ligand polarization tensor or the crystal-field tensor. (The tensor $\mathbf{X}^{(t)}$ may result of the coupling of several $G$-invariant operators according to the order of the mechanism used.) The $C$ coefficients are model-dependent parameters that depend, among other things, on the initial, final and intermediate configurations as well as on the energies of the $p$ photons. Equation (20) unifies various models introduced in [1-4] for $p=1$ and in [5, 13-24] for $p=2$. It can be applied to both (i) parity-allowed and (ii) parity-forbidden transitions.

(i) The particular case of intra-configurational two-photon transitions $(p=2)$ is of special interest. For parity-allowed transitions within the configuration $n \ell^{N}$, it is sufficient to take $t=0$ with $\mathbf{X}^{(0)}=1$ in order to describe second-order mechanisms when $k_{S}=0$ and second- plus third-order mechanisms when $k_{S}=0$ and $k_{S} \neq 0$. The situation where $t \neq 0$ permits to describe (other) third-order mechanisms when $k_{S}=0$ and fourth-order mechanisms when $k_{S} \neq 0$. As a matter of fact, (20) gives back the model introduced by Axe [5] (with $t=0, k_{S}=0$ ) for second-order mechanisms and extended by different authors $[14,15,17,18,20,21]$ (with $t \neq 0, k_{S} \neq 0$ ) to take into account higher-order mechanisms.

(ii) Two particular cases deserve a special attention, viz., the intra-configurational one-photon transitions and the inter-configurational two-photon transitions. For parity- 
forbidden transitions, $\mathbf{X}^{(t)}$ is either the ligand polarization tensor or the odd crystal-field tensor. In the case of one-photon transitions $(p=1)$ within the configuration $n \ell^{N},(20)$ with $k_{S}=0$ gives as a special case the operator implicitly considered by several people in order to explain intra-configurational one-photon transitions for $d^{N}[1,2]$ or $f^{N}[3,4]$ ions in crystals. In the case of inter-configurational two-photon transitions $(p=2)$ between the configuration $n \ell^{N}$ and the configuration $n \ell^{N-1} n^{\prime} \ell^{\prime}$ with $\ell+\ell^{\prime}$ odd, (20) with $k_{S}=0$ yields as particular cases the operators introduced in connection with either a static coupling mechanism $[13,16,19,22,24]$ when $\mathbf{X}^{(t)}$ is the odd crystal-field tensor or a dynamic coupling mechanism $[19,22]$ when $\mathbf{X}^{(t)}$ is the ligand polarization tensor.

The transition matrix element for the operator (20) may be easily calculated owing to (11). We thus get

$$
\begin{aligned}
& M_{i(\Gamma \gamma) \rightarrow f\left(\Gamma^{\prime} \gamma^{\prime}\right)}=\sum_{\alpha^{\prime} S^{\prime} L^{\prime} J^{\prime} a^{\prime}} \sum_{\alpha S L J a}(-1)^{J-J^{\prime}} c\left(\alpha^{\prime} S^{\prime} L^{\prime} J^{\prime} a^{\prime} \Gamma^{\prime} ; f\right)^{*} c(\alpha S L J a \Gamma ; i) \\
& \sum_{k_{1} k_{2} \cdots k_{p-1}} \sum_{t} \sum_{k_{S} k_{L}} \sum_{k} \sum_{a_{0}} C\left[k_{1} k_{2} \cdots k_{p-1} ; t ; k_{S} k_{L} ; k\right] X_{a_{0} \Gamma_{0} \gamma_{0}}^{(t)} \\
& {[k]^{1 / 2}\left(\alpha^{\prime} S^{\prime} L^{\prime} J^{\prime}\left\|W^{\left(k_{S} k_{L}\right) k}\right\| \alpha S L J\right) \sum_{a^{\prime \prime} \Gamma^{\prime \prime} \gamma^{\prime \prime}} \sum_{b^{\prime \prime}} P_{a^{\prime \prime} \Gamma^{\prime \prime} \gamma^{\prime \prime}}^{\left(k_{p-1}\right)}} \\
& f\left(\begin{array}{ccc}
k & k_{p-1} & t \\
b^{\prime \prime} \Gamma^{\prime \prime} \gamma^{\prime \prime} & a^{\prime \prime} \Gamma^{\prime \prime} \gamma^{\prime \prime} & a_{0} \Gamma_{0} \gamma_{0}
\end{array}\right)^{*} f\left(\begin{array}{ccc}
J & J^{\prime} & k \\
a \Gamma \gamma & a^{\prime} \Gamma^{\prime} \gamma^{\prime} & b^{\prime \prime} \Gamma^{\prime \prime} \gamma^{\prime \prime}
\end{array}\right) \text {, }
\end{aligned}
$$

where we have used the fact that the tensor $\mathbf{X}^{(t)}$ has only $G$-invariant components $X_{a_{0} \Gamma_{0} \gamma_{0}}^{(t)}$ distinguishable by the multiplicity label $a_{0}$. The right hand side of $(22)$ can be expressed in terms of $\bar{f}$ coefficients in view of the connecting formula (arising from the comparison of (6) and (11)) between the $f$ and $\bar{f}$ symbols.

By introducing (22), in terms of $f$ or $\bar{f}$ symbols, into (5) and by using the corollary of 
Section 1, we obtain the intensity formula

$$
S_{i(\Gamma) \rightarrow f\left(\Gamma^{\prime}\right)}=\sum_{\left\{k_{i}\right\}} \sum_{\left\{\ell_{i}\right\}} \sum_{r} \sum_{s} \sum_{\Gamma^{\prime \prime}} I\left[\left\{k_{i}\right\}\left\{\ell_{i}\right\} r s \Gamma^{\prime \prime} ; \Gamma \Gamma^{\prime}\right] \sum_{\gamma^{\prime \prime}} P_{r \Gamma^{\prime \prime} \gamma^{\prime \prime}}^{\left(k_{p}\right)}\left(P_{s \Gamma^{\prime \prime} \gamma^{\prime \prime}}^{\left(\ell_{p-1}\right)}\right)^{*}
$$

(with $1 \leq i \leq p-1$ ), the form of which holds for both parity-allowed and parity-forbidden $p$-photon transitions. The intensity parameters $I$ in (23) are given by

$$
\begin{gathered}
I\left[\left\{k_{i}\right\}\left\{\ell_{i}\right\} r s \Gamma^{\prime \prime} ; \Gamma \Gamma^{\prime}\right]:=\sum_{J^{\prime} a^{\prime}} \sum_{J a} \sum_{\bar{J}^{\prime} \bar{a}^{\prime}} \sum_{\bar{J} \bar{a}} \sum_{k \bar{r}} \sum_{\ell \bar{s}} \\
Y_{k}\left(J^{\prime} a^{\prime} \Gamma^{\prime}, J a \Gamma,\left\{k_{i}\right\} r \bar{r} \Gamma^{\prime \prime}\right) Y_{\ell}\left(\bar{J}^{\prime} \bar{a}^{\prime} \Gamma^{\prime}, \bar{J} \bar{a} \Gamma,\left\{\ell_{i}\right\} s \bar{s} \Gamma^{\prime \prime}\right)^{*} \\
Z\left(J a, J^{\prime} a^{\prime}, k \bar{r}, \bar{J} \bar{a}, \bar{J}^{\prime} \bar{a}^{\prime}, \ell \bar{s} ; \Gamma \Gamma^{\prime} \Gamma^{\prime \prime}\right),
\end{gathered}
$$

where the $Y$ symbol is defined through

$$
\begin{aligned}
& Y_{k}\left(J^{\prime} a^{\prime} \Gamma^{\prime}, J a \Gamma,\left\{k_{i}\right\} r \bar{r} \Gamma^{\prime \prime}\right):=\left([J]\left[\Gamma^{\prime \prime}\right]\right)^{-1 / 2}([k][\Gamma])^{1 / 2}(-1)^{J-J^{\prime}} \sum_{\alpha^{\prime} S^{\prime} L^{\prime}} \sum_{\alpha S L} \sum_{k_{S} k_{L}} \\
& c\left(\alpha^{\prime} S^{\prime} L^{\prime} J^{\prime} a^{\prime} \Gamma^{\prime} ; f\right)^{*} c(\alpha S L J a \Gamma ; i)\left(\alpha^{\prime} S^{\prime} L^{\prime} J^{\prime}\left\|W^{\left(k_{S} k_{L}\right) k}\right\| \alpha S L J\right) \\
& \sum_{t a_{0}} C\left[k_{1} k_{2} \cdots k_{p-1} ; t ; k_{S} k_{L} ; k\right] X_{a_{0} \Gamma_{0} \gamma_{0}}^{(t)} f\left(\begin{array}{ccc}
k & k_{p-1} & t \\
\bar{r} \Gamma^{\prime \prime} \gamma^{\prime \prime} & r \Gamma^{\prime \prime} \gamma^{\prime \prime} & a_{0} \Gamma_{0} \gamma_{0}
\end{array}\right)
\end{aligned}
$$

which does not depend on $\gamma^{\prime \prime}$. The $I$ parameters in (24) can be calculated in an ab initio way or can be considered as phenomenological parameters. In both approaches, the following properties and rules are of central importance.

PROPERTY 1. In the general case, we have the (hermiticity) property

$$
I\left[\left\{\ell_{i}\right\}\left\{k_{i}\right\} \operatorname{sr} \Gamma^{\prime \prime} ; \Gamma \Gamma^{\prime}\right]=I\left[\left\{k_{i}\right\}\left\{\ell_{i}\right\} r s \Gamma^{\prime \prime} ; \Gamma \Gamma^{\prime}\right]^{*}
$$

that ensures that $S_{i(\Gamma) \rightarrow f\left(\Gamma^{\prime}\right)}$ is a real number.

PROPERTY 2. In the case where the group $G$ is multiplicity free, we have the factorization formula

$$
I\left[\left\{k_{i}\right\}\left\{\ell_{i}\right\} r s \Gamma^{\prime \prime} ; \Gamma \Gamma^{\prime}\right]=\chi\left[\left\{k_{i}\right\} r \Gamma^{\prime \prime} ; \Gamma \Gamma^{\prime}\right] \chi\left[\left\{\ell_{i}\right\} s \Gamma^{\prime \prime} ; \Gamma \Gamma^{\prime}\right]^{*},
$$


where the function $\chi$ is defined through

$$
\chi\left[\left\{k_{i}\right\} r \Gamma^{\prime \prime} ; \Gamma \Gamma^{\prime}\right]:=\sum_{J^{\prime} a^{\prime}} \sum_{J a} \sum_{k \bar{r}} Y_{k}\left(J^{\prime} a^{\prime} \Gamma^{\prime}, J a \Gamma,\left\{k_{i}\right\} r \bar{r} \Gamma^{\prime \prime}\right)\left(J^{\prime} a^{\prime} \Gamma^{\prime}+k \bar{r} \Gamma^{\prime \prime} \mid J a \Gamma\right)
$$

which follows from (24) and the factorized form of $Z$ (see (18)).

The number of independent parameters $I$ in the expansion (23) can be a priori determined from the two following selection rules used together with Properties 1 and 2 .

RULE 1. In order to have $S_{i(\Gamma) \rightarrow f\left(\Gamma^{\prime}\right)} \neq 0$, it is necessary that

$$
\Gamma^{\prime \prime} \subset \Gamma^{*} \otimes \Gamma \quad \Gamma^{\prime \prime} \subset\left(k_{p-1}\right) \quad \Gamma^{\prime \prime} \subset\left(\ell_{p-1}\right) \quad \Gamma^{\prime \prime} \subset(k) \quad \Gamma^{\prime \prime} \subset(\ell)
$$

where $\left(k_{p-1}\right),\left(\ell_{p-1}\right),(k)$ and $(\ell)$ are IRC's of the group $O(3)$ and $\Gamma^{\prime *}$ is the complex conjugate of $\Gamma^{\prime}$.

RULE 2. The sum over $\left\{k_{i}\right\}$ and $\left\{\ell_{i}\right\}$ in the intensity formula (23) is partially controlled by the following points. (i) The order of the mechanism used for describing the absorption processus: $k_{p-1}$ and $\ell_{p-1}$ cannot vanish if only $p$-order mechanisms, corresponding to $t=$ $k_{S}=0$, are taken into consideration. (In this case, $k_{p-1}=k_{L}=k$ and $\ell_{p-1}=\ell_{L}=\ell$.) Conversely, $k_{p-1}$ and $\ell_{p-1}$ may be zero if higher-order mechanisms, corresponding to $t \neq 0$ and/or $k_{S} \neq 0$, are introduced. (ii) The nature of the photons: if the $p$ absorbed photons have the same polarization, then $k_{1}\left(\right.$ and $\left.\ell_{1}\right)=0,2$ so that if $k_{i}\left(\right.$ or $\left.\ell_{i}\right)=0$ we have $k_{i+1}($ or $\left.\ell_{i+1}\right)=1$ and $k_{i+2}\left(\right.$ or $\left.\ell_{i+2}\right)=0,2$ when $i \geq 1$.

\section{Discussion and Closing Remarks}

For low values of $k_{p-1}$ and $\ell_{p-1}$, there is no summation on $r$ and $s$, two branching multiplicity labels of type $a$, in the intensity formula $(23)$. (The frequency of $\Gamma^{\prime \prime}$ in $\left(k_{p-1}\right)$ and $\left(\ell_{p-1}\right)$ 
is rarely greater than 1 for $p \leq 2$.) The group-theoretical selection rules $(28)$ impose strong limitations on the summation over $\Gamma^{\prime \prime}$ in (23) once $\Gamma$ and $\Gamma^{\prime}$ are fixed and the range of values of $k$ and $\ell$ is chosen.

The number of independent intensity parameters $I$ in (23) is determined by: (i) the number of photons and their nature (polarization, energy), cf. Rules 1 and 2; (ii) the symmetry group $G$, cf. Rule 1; (iii) the symmetry property (26), cf. Property 1; (iv) the use of $t=0$ and $k_{S}=0$ ( $p$-order mechanism) or $t \neq 0$ and $k_{S} \neq 0$ (higher-order mechanisms), cf. Rule 2; (v) the kind of the (weak-, intermediate- or strong-field) coupling used for the state vectors, cf. (3). Points (i)-(ii) depend on external physical conditions. On the other hand, points (iv) and (v) are model-dependent. In particular, in the case where the $J$-mixing, cf. point (v), can be neglected, a situation of interest for lanthanide ions, the summations on $k$ and $\ell$ in (24) are reduced by the rule $\left|J-J^{\prime}\right| \leq k, \ell \leq J+J^{\prime}$, where $J$ and $J^{\prime}$ are the total angular momenta for the initial and final states, respectively. Similar restrictions apply to $k_{S}$ and $k_{L}$ in $(25)$ if the $S$ - and $L$-mixing are neglected.

The computation, via Equations (18) or (19), (24) and (25), of the $I$ parameters is a difficult task in general. Therefore, they may be considered, at least in a first step, as phenomenological parameters. In this respect, Equations (18) or (19), (24) and (25) should serve as a guide for reducing the number of $I$ parameters. Once the number of independent parameters $I$ in the intensity formula (23) has been determined, we can obtain the polarization dependence of the intensity strength $S_{i(\Gamma) \rightarrow f\left(\Gamma^{\prime}\right)}$ by calculating the tensor products $P_{a^{\prime \prime} \Gamma^{\prime \prime} \gamma^{\prime \prime}}^{(K)}\left(\right.$ with $K=k_{p-1}, \ell_{p-1}$ and $\left.a^{\prime \prime}=r, s\right)$ occurring in Equation (23).

To close this Letter, some remarks are in order. In the particular case $p=1$ and 2 , the Hamiltonian model given by (20) and (21) unifies various models described in the literature 
for rare earth ions and transition-metal ions. The originality of this work rests on the use of symmetry adaptation methods for the chain $S U(2) \supset G^{*}$ in conjunction with a very general Hamiltonian model for describing simultaneous absorption of $p$ photons between Stark levels (rather than between $J$ levels). Nontrivial aspects of symmetry adaptation methods have been taken into account in a quantitative way: factorization à la Wigner-Eckart for the chain $S U(2) \supset G^{*}$, Racah's lemma for the corresponding coupling coefficients and orthogonalitycompleteness of the latter. As a net result, we have obtained the intensity formula (23) where the dynamics appears in the $I$ parameters and the geometry is contained in the $P$ factors describing the polarization dependence. The intensity formula (23) for multi-photon absorption can be extended to multi-photon emission as well as to Rayleigh and Raman scattering modulo some caution with the $C$ parameters in (25). The general form of (23) is also valid for other multi-photon processes, as for example the simultaneous absorption of several photons, certain by electric-dipole absorption and others by magnetic-dipole and/or electric-quadrupole absorption.

A word should be said about previous works on this subject. The subject treated in the present Letter has been touched upon in $[13,21,23-26]$ for $p=2$ and fully developed in the thesis by one of the authors (M.D.) for $p$ arbitrary. The accent has been put here on the intensity, rather than on the transition moment as in some works dealing with twophoton spectroscopy $[13,21]$. Furthermore, this work is concerned with models and their use in connection with symmetry adaptation methods, rather than with qualitative symmetry considerations only. In this respect, our work represents a further important step besides the pioneer works by Inoue and Toyozawa [25], on one hand, and by Bader and Gold [26], on the other hand, where only symmetry considerations, arising from the group $G$ considered as an isolated entity, are introduced in the situation where $p=2$. 


\section{References}

1. Sugano, S., Prog. Theor. Phys. (Kyoto) Suppl. 14, 66 (1960).

2. Griffith, J.S., Mol. Phys. 3, 477 (1960).

3. Judd, B.R., Phys. Rev. 127, 750 (1962).

4. Ofelt, G.S., J. Chem. Phys. 37, 511 (1962).

5. Axe, J.D., Jr., Phys. Rev. 136, A42 (1964).

6. Flato, M., J. Mol. Spectr. 17, 300 (1965).

7. Low, W. and Rosengarten, G., J. Mol. Spectr. 12, 319 (1964).

8. Kibler, M., J. Mol. Spectr. 26, 111 (1968); Int. J. Quantum Chem. 3, 795 (1969).

9. Kibler, M. and Grenet, G., Int. J. Quantum Chem. 29, 11 (1986); 29, 485 (1986).

10. Kibler, M., C. R. Acad. Sc. (Paris) B 268, 1221 (1969).

11. Griffith, J.S., The Irreducible Tensor Method for Molecular Symmetry Groups, PrenticeHall, Englewood Cliffs, New Jersey, 1962.

12. Tang Au-chin and coll., Theoretical Method of the Ligand Field Theory, Science Press, Beijing, 1979.

13. Apanasevich, P.A., Gintoft, R.I., Korolkov, V.S., Makhanek, A.G. and Skripko, G.A., Phys. Status Solidi (b) 58 , 745 (1973); Makhanek, A.G., Korolkov, V.S. and Yuguryan, L.A., Phys. Status Solidi (b) 149, 231 (1988).

14. Judd, B.R. and Pooler, D.R., J. Phys. C 15, 591 (1982).

15. Downer, M.C. and Bivas, A., Phys. Rev. B 28, 3677 (1983).

16. Gayen, S.K., Hamilton, D.S. and Bartram, R.H., Phys. Rev. B 34, 7517 (1986).

17. Reid, M.F. and Richardson, F.S., Phys. Rev. B 29, 2830 (1984).

18. Sztucki, J. and Strȩk, W., Phys. Rev. B 34, 3120 (1986).

19. Leavitt, R.C., Phys. Rev. B 35, 9271 (1987).

20. Smentek-Mielczarek, L. and Hess, B.A., Jr., Phys. Rev. B 36, 1811 (1987).

21. Kibler, M. and Gâcon, J.-C., Croat. Chem. Acta 62, 783 (1989).

22. Sztucki, J. and Strȩk, W., Chem. Phys. 143, 347 (1990).

23. Kibler, M.R., in Symmetry and Structural Properties of Condensed Matter, W. Florek, T. Lulek and M. Mucha, eds., World Scientific, Singapore, 1991, p. 237.

24. Daoud, M. and Kibler, M., J. Alloys and Compounds 193, 219 (1993).

25. Inoue, M. and Toyozawa, Y., J. Phys. Soc. Japan 20, 363 (1965).

26. Bader, T.R. and Gold, A., Phys. Rev. 171, 997 (1968). 